Coordination Chemistry

\title{
Surface-Assisted Assembly of 2D Metal-Organic Networks That Exhibit Unusual Threefold Coordination Symmetry**
}

\author{
Sebastian Stepanow, Nian Lin, * Dietmar Payer, Uta Schlickum, Florian Klappenberger, \\ Giorgio Zoppellaro, Mario Ruben, Harald Brune, Johannes V. Barth, and Klaus Kern
}

Coordination-based supramolecular chemistry, ${ }^{[1]}$ with its characteristic control of the self-assembly process and intrinsic defect tolerance, has been proven to be a very efficient synthetic tool to fabricate metallosupramolecular networks of well-defined topology in one, two, and three dimensions. ${ }^{[2-8]}$ This strategy was recently applied to low dimensions by assembling regular molecular architectures from organic molecules and transition-metal centers directly on solid surfaces. ${ }^{[9,10]}$ A variety of surface-supported molecular network structures has been made accessible by the general application of a surface-assisted metal-coordination method to metal centers and aromatic polycarboxylic acids on metal surfaces. ${ }^{[9]}$ As valid for supramolecular structures in general, the structures of the two-dimensional metal-organic coordination networks (2D-MOCNs) formed are predetermined by the properties of the ligands (e.g., donor atoms and their spatial arrangement, steric crowding) and the electronic characteristics of the metal ions (e.g., involved orbitals, ionization energies). However, under 2D conditions, the realization of a given coordination algorithm might be altered by the presence of a metal substrate, which results in deviating coordination geometries for the same metal-ligand coupling in comparison to the 3D situation (e.g., in the bulk phase). Such deviation can be attributed to charge transfer or screening effects and the strict 2D confinement of ligands and metal centers imposed by the substrate, which substantially influences the characteristics of the metal-to-ligand bonding within the $2 \mathrm{D}$ coordination network. ${ }^{[10 \mathrm{e}]}$

[*] Dr. S. Stepanow, Dr. N. Lin, D. Payer, Prof. K. Kern Max-Planck-Institut für Festkörperforschung Heisenbergstrasse 1, 70569 Stuttgart (Germany) Fax: $(+49) 711-6891662$

E-mail:n.lin@fkf.mpg.de

Dr. U. Schlickum, Dr. F. Klappenberger, Prof. H. Brune,

Prof. J. V. Barth, Prof. K. Kern

Institut de Physique des Nanostructures

Ecole Polytechnique Fédérale de Lausanne

1015 Lausanne (Switzerland)

Dr. G. Zoppellaro, Dr. M. Ruben

Institut für Nanotechnologie, Forschungszentrum Karlsruhe 76021 Karlsruhe (Germany)

Prof. J. V. Barth

Departments of Chemistry and Physics \& Astronomy

The University of British Columbia

Vancouver, BC V6T 1Z4 (Canada)

$[* * *$ This work was supported partially by the EC-FP VI STREP "BIOMACH" (NMP4-CT-2003-505-487) and by the ESF-EUROCORES-SONS project "FunSMARTs".
A study of surface-assisted coordination of iron and cobalt centers with polycarboxylic acids has revealed orthogonally arranged 2D-MOCNs exhibiting even, mostly fourfold, symmetry. In contrast, 2D-MOCNs exhibiting threefold symmetry, such as honeycomb structures or similar ones with more complexity, such as kagomé lattices, have not yet been observed on surfaces. Also in the 3D bulk phase, metalorganic coordination network structures that exhibit an oddnumbered symmetry are much less frequently observed than their even-symmetry counterparts. ${ }^{[1]}$ However, a series of 2D hexagonal supramolecular structures was constructed through multiple hydrogen-bonding interactions on different surfaces by using specifically designed organic molecules. ${ }^{[12]}$

Herein we report two surface-supported 2D-MOCNs that exhibit threefold symmetry. Extended hexagonal coordination networks are formed either from iron centers with linear 4,4'-biphenol ligands (1) or from cobalt centers with linear $1,4^{\prime} ; 4^{\prime}, 1^{\prime \prime}$-terphenyl-4,4' $4^{\prime \prime}$-dicarbonitrile ligands (2) on copper or silver single-crystal surfaces. We have shown by using

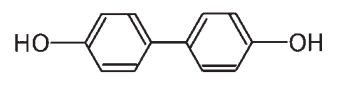

1

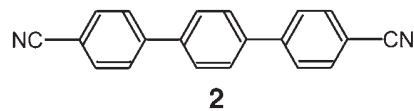

substrates with different symmetries that the threefold symmetry is intrinsic to the metal-ligand coordination and is not due to geometrical templating effects of the underlying surfaces.

STM topographs of the coordination node of the network formed by $\mathrm{Fe}$ centers and three ligands 1 on an $\mathrm{Ag}(111)$ surface and a $\mathrm{Cu}(100)$ surface are shown in Figure $1 \mathrm{a}$ and Figure 1b, respectively. Both reveal a local threefold symmetry, which implies one iron center and three ligands. A model is shown in Figure 1c, in which we assume that under the experimental conditions the molecules of ligand $\mathbf{1}$ are adsorbed with the aromatic rings parallel to the surface plane and that there is no distortion of molecules in comparison with those of the gas phase. Furthermore, X-ray photoemission-spectroscopy measurements reveal that the phenol groups are deprotonated resulting in negatively charged phenolates. ${ }^{[13]}$ The deprotonation process is induced by the catalytic activity of the metallic surfaces and the deposited Fe, which was also observed in studies with moreacidic aromatic polycarboxylic acids. ${ }^{[14-16]}$ The $\mathrm{Fe}-\mathrm{O}$ bond lengths amount to $(2.0 \pm 0.3) \AA$ and the estimated O-Fe-O angles vary from 100 to $140^{\circ}$, depending on the overall network structures. However, with respect to the underlying substrate, the exact positions of the iron atoms and molecules 

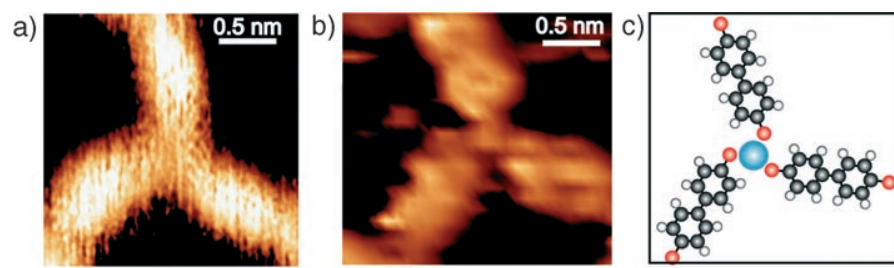

d)
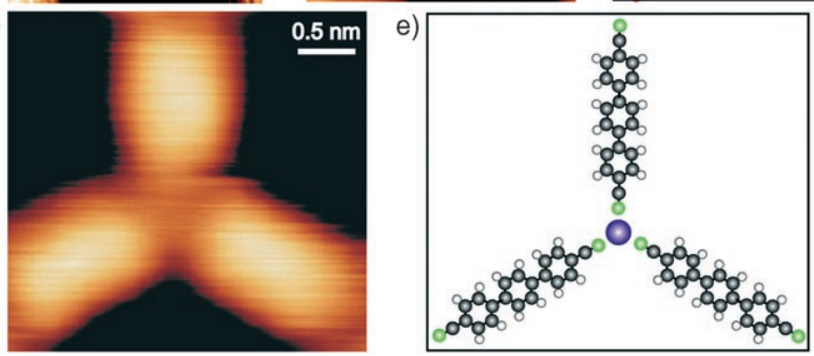

Figure 1. STM topographs of the threefold $\mathrm{Fe}$ (biphenolate) ${ }_{3}$ network node on a) an $\mathrm{Ag}(111)$ surface, and b) a $\mathrm{Cu}(100)$ surface. c) Model of the threefold binding of the nodal Fe(biphenolate) ${ }_{3}$ motif. d) STM topograph of the threefold $\mathrm{Co}$ (dicarbonitrile) ${ }_{3}$ network node on an $\mathrm{Ag}(111)$ surface. e) Model of the threefold binding of the nodal $\mathrm{Co}$ (dicarbonitrile) ${ }_{3}$ motif. For (a) and (b) the image was taken at a tunnel current of $0.1 \mathrm{nA}$ and a bias voltage of $0.5 \mathrm{~V}$, for (d) a tunnel current of $0.3 \mathrm{nA}$ and a bias voltage of $1.9 \mathrm{~V}$. $\mathrm{C}$ gray; $\mathrm{H}$ white; $\mathrm{O}$ red; $\mathrm{N}$ green; Fe light blue; Co dark blue.

1 remain unknown as simultaneous resolution of the atomic lattice of the surfaces was not achieved in our measurements. The STM data elucidate that the ligands do not point directly towards the central $\mathrm{Fe}$ atom but rather tilt clockwise $(R)$ or anticlockwise $(S)$ which accounts for the 2D chirality. ${ }^{[17]}$

A second example of threefold coordination, the coordination of cobalt with 2 on an $\operatorname{Ag}(111)$ surface, is shown in Figure $1 \mathrm{~d}$. The STM data imply that a mononuclear Co center is coordinated by three carbonitrile groups of $\mathbf{2}$, as shown by the model in Figure 1e. The N-Co-N angles are close to the ideal value of $120^{\circ}$. In contrast to the $\mathrm{Fe}$-phenolate coordination, the three linear carbonitrile ligands point symmetrically to the central Co atom. Therefore, the resulting coordination node does not exhibit any chirality.

In $3 \mathrm{D}$ bulk phases, the coordination of iron centers to phenolates leads exclusively to six-, five-, or fourfold coordination motifs; ${ }^{[18]}$ to our knowledge, threefold coordination is not yet known. Similarly, benzonitrile and acetonitrile complexes of a multitude of d- and f-block metals are well-known and have been investigated, ${ }^{[19]}$ but purely threefold coordination has not yet been observed. The emergence of unusual coordination geometries and coordination numbers in this study can be attributed to the very peculiar $2 \mathrm{D}$ environment of the metal surfaces not encountered in the conventional 3D coordination. The substrates cause rehybridization of the orbitals of the metal centers in the surface-supported MOCNs ${ }^{[9 e]}$ which leads to unusual redox states for these metal centers. The tendency of the mostly $\pi$ aromatic ligands to adopt a flat configuration at the surface can stabilize unusual coordination modes. Furthermore, the absence of solvent and gas molecules, which in bulk synthesis are very often able to occupy open coordination sites, may result in the formation of coordinatively unsaturated complexes under ultrahigh vacuum (UHV) conditions.

Figure 2 a displays the overall network structure of the $\mathrm{Fe}-\mathbf{1}$ system developed on an $\mathrm{Ag}(111)$ surface. Two types of hexagons, which are indicated by $T$ and $C$ in Figure $2 \mathrm{a}$, can be detected. The $T$-type hexagons have $C_{3}$ symmetry, while the $C$-type hexagons are compressed in one direction. Both types of hexagon lead to a nearest-neighbor Fe-Fe separation of
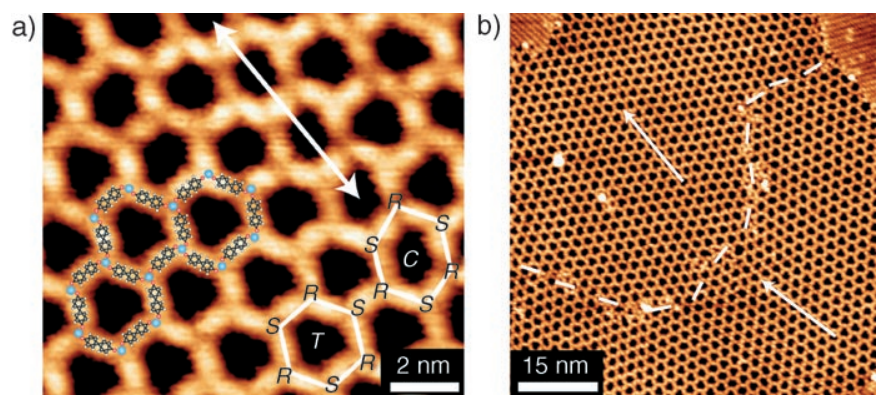

Figure 2. STM topographs displaying the hexagonal Fe-biphenolate network assembled on $\mathrm{Ag}(111)$. a) A high-resolution STM image. A tentative model is superimposed on the data with the same color code as that used in Figure 1. The $T$-type and $C$-type hexagons are highlighted in white frames. The double arrow indicates a ribbon of hexagons only consisting of $C$ hexagons. $R$ and $S$ denote the clockwise and anticlockwise folding, respectively, of the three biphenolate ligands 1 around each iron center. b) An overview showing two domains that are separated by a domain boundary (dashed line). The white arrows mark the domain orientations. The image was taken at a tunnel current of $0.1 \mathrm{nA}$ and a bias voltage of $0.5 \mathrm{~V}$.

approximately $13 \AA$. The O-Fe-O angles of the $T$ hexagons amount to $120^{\circ}$, as expected for a perfect honeycomb. For the $C$ cavity, the $\mathrm{O}-\mathrm{Fe}-\mathrm{O}$ angles deviate by about $20^{\circ}$ from the ideal value of $120^{\circ}$. Both the $T$-type and the $C$-type hexagons have alternately arranged $R$ and $S$ nodes (-RSRSRS-), as shown in Figure $2 \mathrm{a}$. The overall ratio of the two enantiomeric chiral centers is balanced, thus resulting globally in a $2 \mathrm{D}$ racemate.

The network domains can grow continuously over the entire terraces of the $\mathrm{Ag}(111)$ surface and frequently exceed $100 \mathrm{~nm}$. Figure $2 \mathrm{~b}$ shows two domains with different orientations (indicated by two arrows), which enclose an angle of $(14.5 \pm 1)^{\circ}$. The domain boundary (marked by the dashed line) comprises distorted hexagons as well as pentagonheptagon lattice faults. The $C$ hexagons tend to align in straight rows that go through a single domain, as indicated by the arrow in Figure 2 a. At relatively low coverage, when the surface is not yet fully occupied by the network, the $T$-type hexagons predominate. The $C$-type hexagons evolve when the network coverage is close to a full adlayer. Clearly, the lack of open space favors a compression of the network structure.

Figure 3 shows the hexagonal Co-2 network on an $\mathrm{Ag}(111)$ surface. The formation of highly symmetric hexagons is predominant in this network as a consequence of the symmetric achiral coordination nodes. In each of the hexagons formed, the separation between two oppositely faced Co atoms is about $38 \AA$ and a nearest-neighbor distance between Co atoms is about $19 \AA$. 


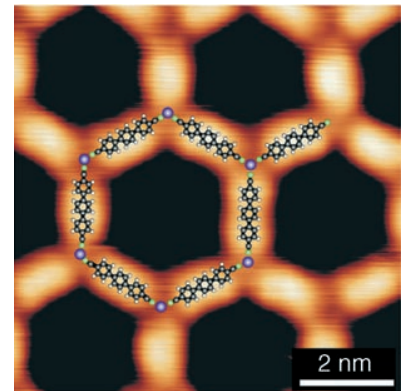

Figure 3. A high-resolution STM image of the honeycomb network of $\mathrm{Co}-\mathbf{2}$ on $\mathrm{Ag}(111)$. A tentative model is superimposed onto the data with the same color code as that used in Figure 1. The image was taken at a tunnel current of $0.3 \mathrm{nA}$ and a bias voltage of $1.9 \mathrm{~V}$.

Possible templating effects of the substrate by assembling the same Fe-biphenolate network on a $\mathrm{Cu}(100)$ surface that exhibits fourfold surface symmetry is discussed below. Figure 4 reveals that hexagonal networks are formed despite
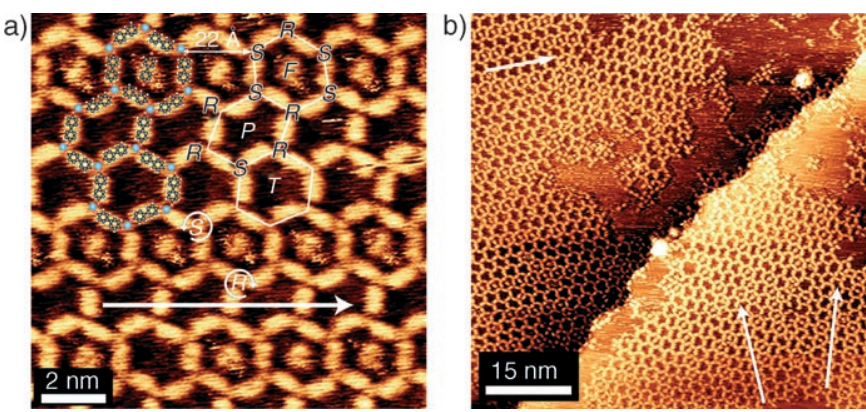

Figure 4. STM topographs of the hexagonal Fe-biphenolate network assembled on $\mathrm{Cu}(100)$. a) A high-resolution STM image of the network. The arrow marks the direction of the high-symmetry row. The white polygons highlight the hexagon frame of the $P$-type, $F$-type, and $T$-type. A tentative model is superimposed on the STM data with the same color code as that used in Figure 1. b) An overview showing three differently oriented domains (shown by the white arrows). The domain size is considerably smaller on $\mathrm{Cu}(100)$ than on $\mathrm{Ag}(111)$. The image was taken at a tunnel current of $0.1 \mathrm{nA}$ and a bias voltage of $0.5 \mathrm{~V}$.

the mismatch between the substrate and the network nodal symmetry. Three types of hexagons are observed: The first type, denoted as $T$ type, shows $C_{3}$ symmetry, while the other two types reveal a low-symmetry parallelogram-like envelope, as indicated by $F$ and $P$ in Figure 4 a. The $\mathrm{O}-\mathrm{Fe}-\mathrm{O}$ angles deviate from the ideal of $120^{\circ}$ and fall in a range between 100 and $140^{\circ}$. Single molecules of $\mathbf{1}$ may be trapped in cavities, primarily in the hexagons of the $F$ type ( $74 \%$ filling rate). The three types of hexagons are present in approximately the same abundance.

Although the network extends in two dimensions, a strict periodicity of $22 \AA$ is only obeyed in one direction (indicated by the straight arrow in Figure $4 \mathrm{a}$ ). This direction is denoted as the high-symmetry row, which consists of a single type of hexagon. Overall we observed four different high-symmetry row orientations (three are present and marked out in the overview STM topograph of Figure 4b), which are related to each other by rotation and mirror symmetry operations of the $\mathrm{Cu}(100)$ surface-symmetry point groups. The domain size of the networks is substantially smaller than for those formed at the $\operatorname{Ag}(111)$ surface. As shown in Figure 4b the distorted hexagons ( $F$ and $P$ types) are formed in the presence of open surface area, in contrast to the formation of the $C$-type hexagons at the $\operatorname{Ag}(111)$ surface, which only form when the adlayer is close to being full. Thus, this distortion is not caused by compression but rather by substrate template effects.

Despite the identical hexagonal topology, the networks formed on the fourfold $\mathrm{Cu}(100)$ surface differ from those formed on the threefold $\mathrm{Ag}(111)$ surface in: 1) the shape and organization of the hexagons; 2) the occurrence of different rotational and mirror domains; 3 ) the size of the single domains. These differences can be explained by template effects of the respective substrates, which cause slight displacements of the network elements from the ideal hexagonal-network positions as a result of the preferred adsorption sites of the molecules and iron atoms at the atomic lattice of the substrate. Because the nodes of a perfect hexagonal network cannot adsorb at the same lattice sites of the fourfold $\mathrm{Cu}(100)$ substrate, the network is deformed and forms the $F$ - and $P$-type hexagons to optimize the adsorption.

Compared with the previously reported hydrogen-bonded nanometer-scale $2 \mathrm{D}$ hexagonal structures,${ }^{[12]}$ the metal-coordination-based hexagonal networks presented herein have several advantages: 1) higher thermal stability arising from the relatively strong coordination bonds; 2) structural flexibility, in particular that the bonding angles may deviate from $120^{\circ}$, which enables the network formation at substrates with different symmetry; 3) specific bonding, which reduces the frequently occurring structural faults in the hydrogen-bonded systems.

In conclusion, for the first time, two 2D-MOCNs expressing hexagonal topologies according to a specific local threefold coordination mode were formed on surfaces. The symmetry of the evolving coordination networks is independent of the symmetry of the substrate, which indicates that the metal-ligand coordination predominates over the substrate influences. Current work addresses the elucidation of the coordination characteristics of the metal centers involved, which will also set the basis for the investigation of the physical properties (e.g., magnetic properties) of the networks formed.

\section{Experimental Section}

Metal atoms $(\mathrm{Fe}, \mathrm{Co})$ and organic molecules $\mathbf{1}$ and $\mathbf{2}$ were subsequently deposited on the metal surfaces in standard UHV chambers with base pressures of less than $2 \times 10^{-10}$ mbar. The $\mathrm{Cu}(100)$ and $\mathrm{Ag}(111)$ surfaces were prepared by repeated cycles of $\mathrm{Ar}^{+}$ion sputtering and subsequent annealing at $800 \mathrm{~K}$, by which flat terraces of up to $100 \mathrm{~nm}$ width separated by monatomic steps were obtained. Ligand 1 was bought (Fluka Chemie $\mathrm{GmbH}$, Germany; purity $>98 \%$ ) and ligand $\mathbf{2}$ was synthesized following a modified procedure of a given protocol. ${ }^{[20]}$ Both compounds were deposited by organic molecular beam epitaxy (OMBE) from a Knudsen cell evaporator, held at 415 (1) or $460 \mathrm{~K}$ (2) during deposition. Fe/Co atoms were evaporated by using an evaporator heated with an electron beam and 
a flux in the range of a few percent of a monolayer per minute. Deposition of $\mathbf{1}$ and Fe was performed at a substrate temperature of $400 \mathrm{~K}$ and deposition of $\mathbf{2}$ and Co at a substrate temperature of $300 \mathrm{~K}$. Coverage below full monolayer saturation was employed for the molecular precursor layers to leave space for the formation of the more-open network structures. STM experiments with constantcurrent mode were performed in situ after samples had been cooled to room temperature or $10 \mathrm{~K}$.

Received: September 6, 2006

Published online: December 8, 2006

Keywords: coordination modes - ligand effects . scanning probe microscopy - supramolecular chemistry . surface chemistry

[1] J. M. Lehn, Supramolecular Chemistry: Concepts and Perspectives, VCH, Weinheim, 1995.

[2] S. Leininger, B. Olenyuk, P. J. Stang, Chem. Rev. 2000, 100, $853-$ 908.

[3] B. J. Holliday, C. A. Mirkin, Angew. Chem. 2001, 113, $2076-$ 2098; Angew. Chem. Int. Ed. 2001, 40, 2022-2043, and references therein.

[4] M. Ruben, J. Rojo, F. J. Romero-Salguero, L. H. Uppadine, J.-M. Lehn, Angew. Chem. 2004, 116, 3728-3747; Angew. Chem. Int. Ed. 2004, 43, 3644-3662.

[5] a) B. Moulton, M. J. Zaworotko, Chem. Rev. 2001, 101, 1629, and references therein; b) S. Kitagawa, R. Kitaura, S. Noro, Angew. Chem. 2004, 116, 2388-2430; Angew. Chem. Int. Ed. 2004, 43 2334-2375, and references therein; c) O. M. Yaghi, M. O'Keeffe, N. W. Ockwig, H. K. Chae, M. Eddaoudi, J. Kim, Nature 2003, 423, 705, and references therein.

[6] J. Michl, T. F. Magnera, Proc. Natl. Acad. Sci. USA 2002, 99, 4788.

[7] A. Semenov, J. P. Spatz, M. Möller, J.-M. Lehn, B. Sell, D. Schubert, C. H. Weidl, U. S. Schubert, Angew. Chem. 1999, 111, 2701-2705; Angew. Chem. Int. Ed. 1999, 38, 2547-2550.

[8] D. G. Kurth, N. Severin, J. P. Rabe, Angew. Chem. 2002, 114, 3833-3835; Angew. Chem. Int. Ed. 2002, 41, 3681-3683.

[9] a) A. Dmitriev, H. Spillmann, N. Lin, J. V. Barth, K. Kern, Angew. Chem. 2003, 115, 2774-2777; Angew. Chem. Int. Ed. 2003, 42, 2670-2673; b) M. A. Lingenfelder, H. Spillmann, A. Dmitriev, S. Stepanow, N. Lin, J. V. Barth, K. Kern, Chem. Eur. J. 2004, 10, 1913-1919; c) S. Stepanow, M. A. Lingenfelder, A Dmitriev, H. Spillmann, E. Delvigne, N. Lin, X. Deng, C. Cai, J. V. Barth, K. Kern, Nat. Mater. 2004, 3, 229-233; d) N. Lin, S. Stepanow, F. Vidal, J. V. Barth, K. Kern, Chem. Commun. 2005, 1681-1683; e) A. P. Seitsonen, M. A. Lingenfelder, H. Spillmann, A. Dmitriev, S. Stepanow, N. Lin, K. Kern, J. V. Barth, J. Am. Chem. Soc. 2006, 128, 5634-5635; f) S. Clair, S. Pons, S Fabris, S. Baroni, H. Brune, K. Kern, J. V. Barth, J. Phys. Chem. $B$ 2006,110, 5627-5632; g) T Classen, G. Fratesi, G. Costantini, S. Fabris, F. L. Stadler, C. Kim, S. de Gironcoli, S. Baroni, K. Kern, Angew. Chem. 2005, 117, 6298-6301; Angew. Chem. Int. Ed. 2005, 44, 6142-6145; h) S. Stepanow, N. Lin, J. V. Barth, K. Kern, J. Phys. Chem. B. 2006, 110, 23472-23477

[10] a) M. Ruben, Angew. Chem. 2005, 117, 1620-1623; Angew. Chem. Int. Ed. 2005, 44, 1594-1596; b) N. Lin, S. Stepanow, F.
Vidal, K. Kern, S. Alam, S. Strömsdörfer, S. Dremov, P. Müller, A. Landa, M. Ruben, Dalton Trans. 2006, 2794-2800.

[11] a) L. R. Macgillivray, S. Subramanian, M. J. Zaworotko, J. Chem. Soc. Chem. Commun. 1994, $1325-1326$; b) X. Xu, M. Nieuwenhuyzen, S. L. James, Angew. Chem. 2002, 114, 790-793; Angew. Chem. Int. Ed. 2002, 41, 764-767; c) A. B. Mallik, S. Lee, E. B. Lobkovsky, Cryst. Growth Des. 2005, 5, 609.

[12] a) J. A. Theobald, N. S. Oxtoby, M. A. Phillips, N. R. Champness, P. H. Beton, Nature 2003, 424, 1029-1031; b) S. J. H. Griessl, M. Lackinger, F. Jamitzky, T. Markert, M. Hietschold, W. M. Heckl, J. Phys. Chem. B 2004, 108, 11556-11560; c) S. De Feyter, A. Gesquière, M. Klapper, K. Müllen, F. C. De Schryver, Nano Lett. 2003, 3, 1485 -1488; d) H.-J. Yan, J. Lu, L.-J. Wan, C.-L. Bai, J. Phys. Chem. B 2004, 108, 11251-11255; e) M. Stöhr, M. Wahl, C. H. Galka, T. Riehm, T. A. Jung, L. H. Gade, Angew. Chem. 2005, 117, 7560-7564; Angew. Chem. Int. Ed. 2005, 44, 73947398; f) N. Lin, D. Payer, A. Dmitriev, T. Strunskus, C. Wöll, J. V. Barth, K. Kern, Angew. Chem. 2005, 117, 1512-1515; Angew. Chem. Int. Ed. 2005, 44, 1488-1491; g) M. Ruben, D. Payer, A. Landa, A. Comisso, C. Gattinoni, N. Lin, J.-P. Collins, J.-P. Sauvage, A. De Vita, K. Kern, J. Am. Chem. Soc. 2006, DOI:10.1021/ja063601k.

[13] a) X.-C. Guo, R. J. Madix, Surf. Sci. 1995, 341, L1065-L1071; b) S. Stepanow, T. Strunskus, N. Lin, C. Wöll, K. Kern, unpublished results.

[14] S. Stepanow, T. Strunskus, M. Lingenfelder, A. Dmitriev, H. Spillmann, N. Lin, J. V. Barth, C. Wöll, K. Kern, J. Phys. Chem. B 2004, 108, 19392.

[15] a) B. G. Frederick, M. R. Ashton, N. V. Richardson, T. S. Jones, Surf. Sci. 1993, 292, 33; b) S. Poulston, R. A. Bennett, A. H. Jones, M. Bowker, Phys. Rev. B 1997, 55, 12888; c) M. Bowker, S. Haq, R. P. Holroyd, P. M. Parlett, S. Poulston, N. Richardson, J. Chem. Soc. Faraday Trans. 1996, 92, 4683; d) A. Dmitriev, N. Lin, J. Weckesser, J. V. Barth, K. Kern, J. Phys. Chem. B 2002, 106, 6907-6912.

[16] a) N. Lin, A. Dmitriev, J. Weckesser, J. V. Barth, K. Kern, Angew. Chem. 2002, 114, 4973-4977; Angew. Chem. Int. Ed. 2002, 41, 4779-4783; b) N. Lin, D. Payer, A. Dmitriev, T. Strunskus, C. Wöll, J. V. Barth, K. Kern, Angew. Chem. 2005, 117, 1512-1515; Angew. Chem. Int. Ed. 2005, 44, 1488-1491.

[17] a) P. Messina, A. Dmitriev, N. Lin, H. Spillmann, M. Abel, J. V. Barth, K. Kern, J. Am. Chem. Soc. 2002, 124, 14000; b) A. Dmitriev, H. Spillmann, M. Lingenfelder, N. Lin, J. V. Barth, K. Kern, Langmuir 2004, 20, 4799.

[18] a) M. R. A. Blomberg, P. E. M. Siegbahn, Theor. Chem. Acc. 1997, 97, 72-80; b) S. Kimura, E. Bill, E. Bothe, T. Weyhermüller, K. Wieghardt, J. Am. Chem. Soc. 2002, 124, 6025-6039; c) B. Sieklucka, R. Podgajny, P. Przychodzen, T. Korzeniak, Coord. Chem. Rev. 2005, 249, 2203-2221.

[19] a) M. H. Garcia, P. J. Mendes, A. R. Dias, J. Organomet. Chem. 2005, 690, 4063-4071; b) L. J. Moitsheki, S. A. Bourne, L. R. Nassimbeni, Acta Crystallogr. Sect. E 2005, 61, m2580-m2581; c) A. W. Zanella, K. H. Ford, P. C. Ford, J. Am. Chem. Soc. 1978, 100,1051-1054; d) A. E. Enriquez, B. L. Scott, M. P. Neu, Inorg. Chem. 2005, 44, 7403-7413; e) C. J. Siemer, M. J. Van Stipdonk, P. K. Kahol, D. M. Eichhorn, Polyhedron 2004, 23, 235-238; f) T. Murahashi, T. Nagai, T. Okuno, T. Matsutani, H. Kurosawa, Chem. Commun. 2000, 1689-1690.

[20] J. Colonge, Bull. Soc. Chim. Fr. 1967, 4370-4374. 